\title{
Differentiation of spontaneous locomotor activity affects the spatial reference memory level in Water Morris test in rats
}

Joanna Dunacka, Justyna Marchewka, Grzegorz Świątek, Marcin Wyroślak, Mariola Grałek, Danuta Wrona

Department of Animal and Human Physiology, Faculty of Biology, University of Gdańsk, Wita Stwosza 59 Street, $80-308$ Gdańsk, Poland

Correspondence: joanna.dunacka@phdstud.ug.edu.pl

DOI: $10.31708 /$ spi1.2018.1

\section{Introduction}

Novelty test, used as a model of stress reactivity (sensitivity/resistance to stress), allows to divide animals according to their level of locomotor response to novelty into high (HRs-) and low (LRs-) responders. These behavioral differences reflect a number of differences, including the level of anxiety, which could affect memory processes and learning. The presented study aimed to determine the effect of differences in spontaneous locomotor activity on the level of spatial reference memory in basal conditions in rats which will be exposed to a sporadic form of Alzheimer's disease (SAD)-induced cognitive impairment in the next stage of the experiment.

Keywords: rats, Alzheimer's disease, novelty test, Water Morris test, spatial reference memory.

\section{Materials and Methods}

Twenty male Wistar rats divided in novelty test (4-6 p.m) into HR/LR animals based on the median for their horizontal movements. Then, spatial reference memory was examined using the Water Maze test that was conducted for 4 days. For the first three days, each rat made four attempts to find a platform (maximum time 120s) per day with 10 minutes apart, and the platform was in a permanent place at that time. On the 4th day (probe test), the platform was removed. This allowed determining the latency of reaching the place where the platform should be and the percentage of staying in the right quadrant that measure of the level of spatial reference memory.

\section{Results}

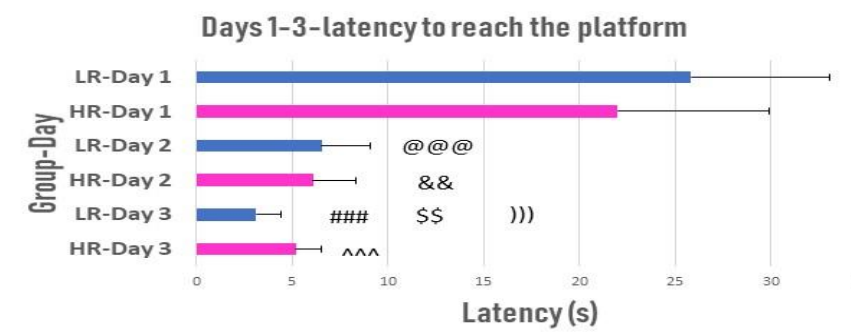

Probe test-latency to reach the place where the platform was present before

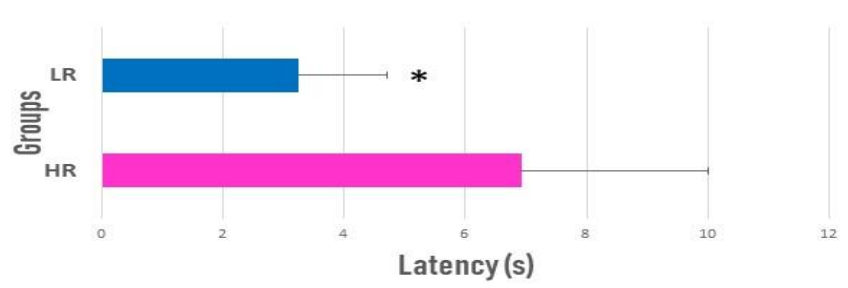

Probe test-latency to reach the quadrant where the platform was present before

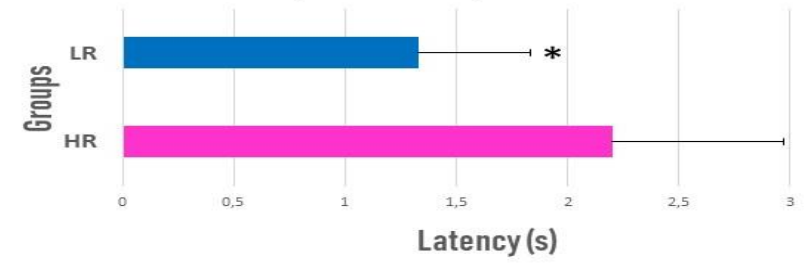

Legend:

Results (Mann-Whitney test) are expressed as mean $+/$ - SD ${ }^{*} \mathrm{p}<0,05$ as compared to HR @@@ $\mathrm{p}<0,001$ as compared to LR-Day 1

$\& \& p<0,01$ as compared to HR-Day 1 \#\#\# $\mathrm{p}<0,001$ as compared to LR-Day 1

$\$ \$ p<0,01$ as compared to HR-Day 3

))) $p<0,001$ as compared to LR-Day 2

$\wedge \wedge \wedge p<0,001$ as compared to HR Day 2 


\section{Discussion}

As far as we know, there is no study on the influence of HR/LR differences on the memory process in rats which will be exposed to streptozotocin-induced SAD. Our previous results (Majkutewicz et al., 2016, 2018) demonstrated impairment of memory and learning in rats with the SAD model that were exposed to the Water Maze test. Neurodegeneration and central inflammatory process supported these cognitive disturbances. The present results suggest that LR rats, that differ according to their behavioral/stress response characteristics from HR animals, in baseline (before SAD model was induced) had higher level of spatial reference memory, as indicated by faster learning process (lower latency to reach the platform and quadrant where the platform was present before) measured in Morris water test. According to (Piazza et al. 1989) LR rats are less susceptible to stress (lower corticosterone release during novelty stress) but have a higher level of anxiety that could influence learning and memory process. The obtained results indicate the significance of differences in behavioral/stress reactivity (HR/LR) measured by spontaneous locomotor activity in the level of memory process in the baseline. It suggests that these differences could predict the development of cognitive disorders in rats with the SAD model.

\section{Acknowledgments}

The authors declare that they have no statements and conflict of interest. Funding came from the Ministry of Science and Higher Education of Poland funding for statutory activities of the Department of Animal and Human Physiology of the University of Gdańsk.

\section{References}

Majkutewicz, I., Kurowska, E., Podlacha, M., Myślińska, D., Grembecka, B., Ruciński, J., Plucińska, K., Jerzemowska, G., \& Wrona, D. (2016). Dimethyl fumarate attenuates intracerebroventricular streptozotocin-induced spatial memory impairment and hippocampal neurodegeneration in rats. Behavioural Brain Research, 308, 24-37.

Majkutewicz, I., Kurowska, E., Podlacha, M., Myślińska, D., Grembecka, B., Ruciński, J., Pierzynowska, K., \& Wrona, D. (2018). Age-dependent effects of dimethyl fumarate on cognitive and neuropathological features in the streptozotocin-induced rat model of Alzheimer's disease. Brain Research,1686, 19-33.

Piazza, P.V., Deminiere, J.M., Le Moal, M., \& Simon, H. (1989). Factors that predict individual vulnerability to amphetamine self-administration. Science, 245(4925), 1511-1513. 